\title{
Über Olibanole;
}

von Emil Fromm und Richard Klein.

[Mitteilung aus dem Chemischen Universitätslaboratorium zu Freiburg i. B.]

(Eingelaufen am 8. Januar 1921.)

Pinonsäure ist von v. Baeyer ${ }^{1}$ ) über Pinsäure und Oxypinsäure zur Norpinsäure abgebaut worden. Pinononsäure haben Wagner und Erschtschikowski ${ }^{2}$ ) durch Bromlange in dieselbe Norpinsäure verwandelt. Gegen die Konstitutionsformeln, welche zuletzt von den ge nannten Autoren festgestellt sind, dürften sich Einwände kaum mehr ergeben.<smiles>CC(=O)C1CCCC1C(C)=O</smiles>

Pinonäsure $\mathrm{C}_{10} \mathrm{H}_{16} \mathrm{O}_{3}$

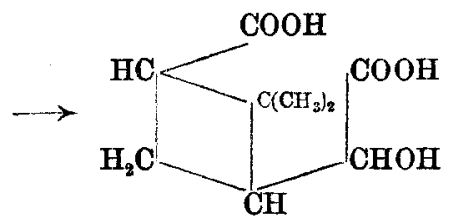

Oxypinsäure $\mathrm{C}_{9} \mathrm{H}_{14} \mathrm{O}_{5}$

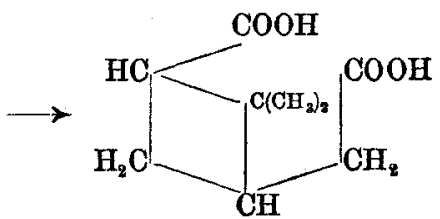

Pinsäure $\mathrm{C}_{9} \mathrm{H}_{14} \mathrm{O}_{4}$

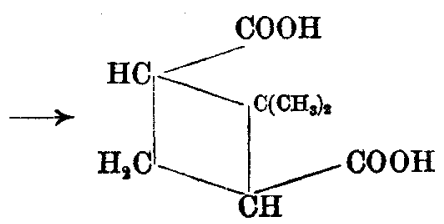

Norpinsäure $\mathrm{C}_{8} \mathrm{H}_{12} \mathrm{O}_{4}$<smiles>CC(C)C(C(=O)O)C(=O)O</smiles>

Pinononsäure $\mathrm{C}_{9} \mathrm{H}_{14} \mathrm{O}_{5}$

1) Ber. d. d. chem. Ges. 29, 1911 (1896).

7) Ber. d. d. chem. Ges. 29, 881 (1896). 
Pinononsäure wurde gewonnen von Wagner und Erschtschikowski ${ }^{1}$ ) durch Permanganatoxydation des Pinens, von Kerschbaum ${ }^{2}$ ) durch Oxydation des Verbenons aus Verbenaöl, von Blumann und Zeitschel ${ }^{3}$ ) durch Oxydation von Verbenon and Verbenol, welche sie unter den Autoxydationsprodukten des Pinens fanden, endlich von Fromm und Autina) durch Abban des Olibanols aus Weihrauchöl. Als Abbauprodukt so verschiedener Naturstoffe ist die Pinononsäure besonders wichtig; um so mehr ist es zu bedauern, dab die Identität der von den verschiedenen Forschern gewonnenen Pinononsäuren nicht mit voller Sicherheit feststeht. Die Säure schmilzt nach Wagner bei $128-129^{\circ}$, nach Kerschbaum bei $127-128^{\circ}$, nach Blumann und Zeitschel in der 1-Modifikation bei $124-128^{\circ}$, in der d-Modifikation bei $128-129^{\circ}$, nach Fromm und Autin bei $130^{\circ}$. Ihr Oxim soll nach Wagner und Erschtschikowski bei $178^{\circ}$ (wahrscheinlich Schreibfehler) schmelzen und schmilzt nach Fromm und A utin bei $187^{\circ}$. Für das Semicarbazon der Säure geben Kerschbaum den Schmelzp. zu $212^{\circ}$, Blumann und Zeitschel zu $204^{\circ}$ und Fromm und Autin zu $209^{\circ}$ an. Fine besondere Schwierigkeit erwächst noch aus dem Umstande, daß das Pinonsäuresemicarbazon, wie sich zeigte, ebenfalls den Schmelzpunkt $204^{\circ}$ zeigt.

So erschien es notwendig, die aus den verschiedenen Ausgangsmaterialien gewonnenen Pinononsäuren miteinander selbst und in ihren Abkömmlingen zu vergleichen. Blumann und Zeitschel haben nun bereits ${ }^{5}$ festgestellt, daß ein von Kerschbaum ihnen überlassenes Präparat von Pinononsäuresemicarbazon aus Verbenon mit dem ibren aus den Antoxydationsprodukten des

1) a. a. 0 .

9) Ber. d. d. chem. Ges. 33, 885 (1900).

s) Ber. d. d. chem. Ges. 46, 1178 (1918).

4) Diese Annalen 401, 253 (1913)-

5) Ber. d. d. chem. Ges. 46, 1183 (1913).

Annalen der Chemie 425. Band. 
Pinens keine Schmelzpunktsdepression ergab and dab demnach beide Präparate identisch sind. Auch ons hat Herr Dr. Kerschbaum liebenswürdigerweise ein kleines Präparat seines Semicarbazons überlassen, genug, um damit festzustellen, daß es auch mit dem Pinononsäuresemicarbazon aus Olibanol von Fromm und Autin keine Depression gibt und dab beide Präparate bei genauer Nachprüfung den von Blumann und Zeitschel angegebenen Schmelzp. $204^{\circ}$ zeigen. Demnach sind alle diese Stoffe identisch. So bleibt nur noch die Pinononsäure übrig, welche Wagner und Erschtschikowski durch Permanganatoxydation von Pinen erhalten hatten. Diese haben wir uns selbst darzustellen versucht und zu diesem Zwecke reines französisches Pinen von der Firma Schimmel \& Co., Siedep. 156-158 $, \mathrm{D}_{18} 0,8683$, sorgfältig auf dem Wasserbade im Vakuum und darauf unter Atmosphärendruck rektifiziert und das so gewonnene Produkt genau nach der Vorschrift von Wagner mit 1 prozentiger kalter Permanganatlösung oxydiert. Es ergab sich bei Wiederholung der Oxydation, daß die Ergebnisse sich nicht ändern, wenn man auch mit 5 prozentiger Permanganatlösung arbeitet. Die Oxydationsprodukte wurden in einen neutralen und einen sauren Teil zerlegt. Der neutrale Teil enthielt noch viel unverändertes Pinen, welches bei weiteren Oxydationen abgebaut werden konnte. Das Gemenge von Säuren wurde mit je einem Äquivalent Semicarbazidchlorhydrat und Natriumacetat behandelt, wobei ein Semicarbazon isoliert wurde, welches den gleichen Schmelzpunkt aufwies wie das Pinononsäuresemicarbazon; nämlich $204^{\circ}$. Dennoch war das so dargestellte Semicarbazon nicht das der Pinononsäure, da es mit reinem Pinononsänecarbazon aus Verbenon von Kerschbaum oder solchem aus Olibanol von Fromm und Autin eine wesentliche Schmelzpunktserniedrigung gab. Seine Zusammensetzung stimmt auf nicht ganz reines Pinonsäuresemicarbazon: 
$0,1380 \mathrm{~g}$ gaben $0,2776 \mathrm{CO}_{2}$ und $0,1064 \mathrm{H}_{2} \mathrm{O}$.

$0,1373 \mathrm{~g} \quad 0,2782 \mathrm{CO}_{2} \Rightarrow 0,1060 \mathrm{H}_{9} \mathrm{O}$.

$0,1402 \mathrm{~g} \quad 222 \mathrm{ccm}$ Stickgas bei $22^{\circ}$ und $744 \mathrm{~mm}$ Druck. $0,1543 \mathrm{~g} \quad, \quad 24,8 \mathrm{cem}$ Stickgas bei $23,5^{\circ}$ und $743 \mathrm{~mm}$ Druck.

\begin{tabular}{ccrr} 
& Ber. für $\mathrm{C}_{11} \mathrm{H}_{18} \mathrm{O}_{3} \mathrm{~N}_{3}$ & \multicolumn{2}{c}{ Gef. } \\
$\mathrm{C}$ & 54,75 & 54,88 & 55,28 \\
$\mathrm{H}$ & $\mathbf{7 , 9 4}$ & 8,63 & 8,64 \\
$\mathrm{~N}$ & 17,3 & 17,6 & 18,09
\end{tabular}

Das neue Semicarbazon wird leicht bei der Behandlung mit verdïnnter Salzsäure gespalten und liefert hierbei ein Gemenge von zwei Säuren, von denen eine bei $100^{\circ}$ schmolz und nur in sehr geringer Menge entsteht, während die andere bei $62^{\circ}$ wie die Pinonsäure schmilzt and anch deren Zusammensetzung hat:

$0,1186 \mathrm{~g}$ gaben $0,2814 \mathrm{CO}_{2}$ und $0,0939 \mathrm{H}_{2} \mathrm{O}$.

$\begin{array}{ccc} & \text { Ber. für } \mathrm{C}_{10} \mathrm{H}_{10} \mathrm{O}_{3} & \text { Gef. } \\ \mathrm{C} & 65,17 & 64,73 \\ \mathrm{H} & 8,76 & 8,86\end{array}$

Da sich das Semicarbazon der Pinononsäure, wie man sich an anderen Versuchen überzengen konnte, auch aus Gemischen sehr leicht ausscheidet, so war hier kein Zweifel, daß bei der Oxydation ganz reinen Pinens Pinononsäure auch nicht in Spuren entstanden sein konnte. Auch die naheliegende Annahme, daß Wagner und Erschtschikowski die Oxydation so lange weiter geführt hätten, bis die Pinonsäure zu Pinononsäure oxydiert war, ist nicht zu halten; denn es ließ sich leicht zeigen, daß Pinonsäure bei der Oxydation mit Permanganatlösung in der Kälte nicht angegriffen wird, auch nach zweimaliger Nachoxydation wurde aus der Permanganatlösung nur unveränderte Pinonsäure als Semicarbazon isoliert.

Da reines Pinen also bei der Permanganatoxydation keine Pinononsäure liefert, so mub die von Wagner und Erschtschikowski erhaltene Säure aus einer Verunreinigung des Pinens abstammen. Welcher Art diese Verunreinigung gewesen sein mag, läßt sich nach den Untersuchungen von Blumann und Zeitschel leicht 
vermuten, nämlich die verbenon- und verbenolhaltigen Autoxydationsprodukte des Pinens.

Dem Verbenon $\mathrm{C}_{10} \mathrm{H}_{14} \mathrm{O}$, welches sich zu Pinononsäure oxydieren läbt, kommt ohne Zweifel die Formel I zu. Die Stoffe $\mathrm{C}_{10} \mathrm{H}_{16} \mathrm{O}$, welche bei der Oxydation Pinononsäure liefern, können nur die Formeln Ia, II, III und IIIa, sowie IV and IV a haben. Verbenol, der Alkohol des Ketóns Verbenon I, muß der Formel Ia entsprechen, Dihydroverbenon, das Hydrierungsprodukt des Verbenons, entspricht demnach der Formel III a. Da III nur die Enolform von IIIa ist, so bleiben nur noch die Formeln II und IV, sowie die Ketoform des letzteren IV a für das Olibanol von Fromm und Autin, welches gleichfalls bei der Oxydation Pinononsäure liefert, übrig, vorausgesetzt, daß sich Olibanol nicht als identisch mit Verbenol oder Dihydroverbenon erweisen sollte.
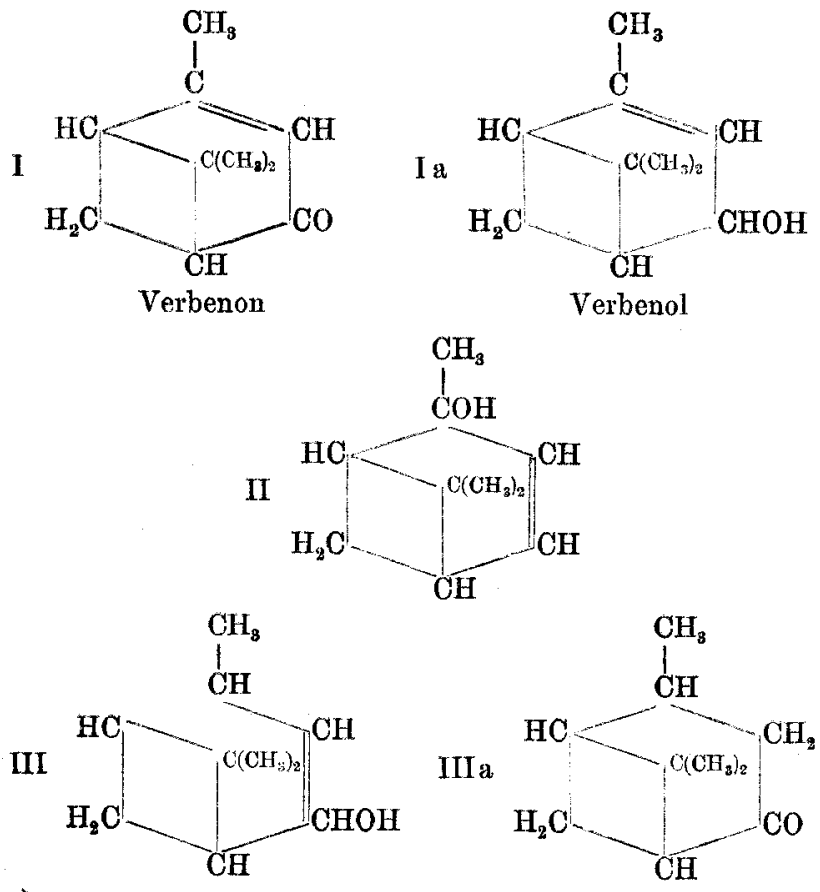

Dihydroverbenon 

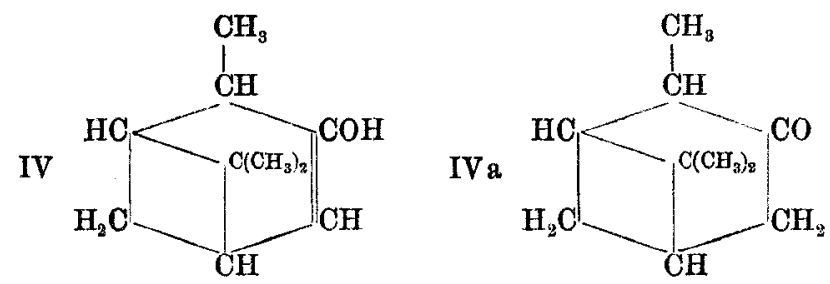

Zur Entscheidung dieser Fragen wurde die Untersuchung des Weihrauchöles wieder aufgenommen und zur Gewinnung von Olibanol $1 \mathrm{~kg}$ terpenfreies Weihranchöl der Firma Schimmel \& Co. in Miltitz der fraktionierten Destillation unterworfen. Diese wurde diesesmal abweichend von dem ehedem von Fromm und Autin angewendeten Verfahren in vacuo durchgeführt, und es gelang leicht, wieder eine Fraktion als rein zu erkennen, welche Siedep. ${ }_{22} 117-119^{\circ}$, Siedep. $210-211^{\circ}$, $\mathrm{D}_{18} 0,9504$ zeigte. Das war augenscheinlich das Olibanol von Fromm und Autin, um so mehr als es die Zusammensetzung $\mathrm{C}_{10} \mathrm{H}_{10} \mathrm{O}$ hatte.

$$
\begin{aligned}
& 0,1185 \mathrm{~g} \text { gaben } 0,3414 \mathrm{CO}_{2} \text { and } 0,1161 \mathrm{H}_{\mathbf{2}} \mathrm{O} . \\
& 0,1078 \mathrm{~g} " \quad 0,3106 \mathrm{CO}_{2} \quad 0,0986 \mathrm{H}_{2} \mathrm{O} .
\end{aligned}
$$

\begin{tabular}{ccrr} 
& Ber. für $\mathrm{C}_{10} \mathrm{H}_{16} \mathrm{O}$ & \multicolumn{2}{c}{ Gef. } \\
$\mathrm{C}$ & $\mathbf{7 8 , 8 8}$ & $\mathbf{7 8 , 6 0}$ & $\mathbf{7 8 , 6 0}$ \\
$\mathrm{H}$ & 10,60 & 10,96 & 10,24
\end{tabular}

Das durch Vakuumdestillation gewonnene Olibanol ließ sich auch wie das von Fromm und Autin mit Permanganat oxydieren und lieferte bei der 0xydation auch saure Produkte, diese enthielten aber erstaunlicherweise keine Spur von krystallisierender Säure, auch ist es auf keine Weise gelungen, mit Semicarbazid ein Semicarbazon daraus abzuscheiden. Kurz, es war überhaupt keine Pinononsäure entstanden. Es zeigte sich bald, daß man das pinononsäureliefernde Olibanol nur dann erhält, wenn man nicht im Vakuum, sondern unter Atmosphärendruck destilliert. Es genügt, das in vacuo erbaltene Produkt einige Male direkt zu destillieren und das Destillat zu oxydieren, um wieder gute Ansbenten an Pinononsäure zu erhalten. Demnach gibt es zwei Oli- 
banole, eines welches sich im natürlichen Weihrauchöl findet und bei der Oxydation keine Pinonsäure liefert - wir nennen es in Zukunft $\boldsymbol{a}$-Olibanol - und eines, welches erst durch Erhitzen über den Siedepunkt durch Umlagerung ans dem Urprodukt entsteht, welches Pinonsäure bei der Oxydation liefert und in Zukanft $\beta$-Olibanol heißen soll.

$\alpha$-Olibanol kann keiner der oben aufgeführten Formeln I-IV a entsprechen, da es bei der Oxydation keine Pinononsäure liefert, ist demnach bestimmt auch weder mit Verbenol noch mit Dihydroverbenon identisch. Mit Natrium reagiert es nur schwach beim Erwärmen, mit Phenylhydrazin, Semicarbazid und Hydroxylamin konnten krystallisierte Produkte bisher nicht gewonnen werden. Auch nach der Oxydation mit Chromsäure war in dem neutralen Produkt eine Carbonylgruppe mit den üblichen Agenzien nicht nachzuweisen. Bei der Oxydation mit Permanganat entsteht, wie bereits erwähnt, keine Pinonsäure, wohl aber eine Säure von honigartiger Konsistenz, welche derjenigen ähnelt, die neben Pinononsäure bei der Oxydation von $\beta$-Olibanol entsteht und der Formel $\mathrm{C}_{10} \mathrm{H}_{16} \mathrm{O}_{4}$ entspricht. Leider konnte aus Materialmangel und wegen der wenig charakteristischen Eigenschaften dieser Säure eine Identifizierung bisher nicht ausgeführt werden.

Reduziert man $\alpha$-Olibanol mit dem 5 fachen der berechneten Menge Natrium in absolutem Alkohol, so entsteht ein Dihydroolibanol $\mathrm{C}_{10} \mathrm{H}_{18} \mathrm{O}$, ein farbloses Öl von salbeiartigem Geruche, Siedep $._{25} 100-102^{\circ}$.

$0,1360 \mathrm{~g}$ gaben $0,3848 \mathrm{CO}_{2}$ und $0,1380 \mathrm{H}_{2} \mathrm{O}$. $0,1370 \mathrm{~g}, \quad 0,3912 \quad \mathrm{CO}_{2}$.

\begin{tabular}{lcccc} 
& Ber. für $\mathrm{C}_{10} \mathrm{H}_{18} \mathrm{O}$ & \multicolumn{3}{c}{ Gef. } \\
$\mathrm{C}$ & 77,85 & 77,19 & & 77,9 \\
$\mathrm{H}$ & 11,75 & & 11,35 &
\end{tabular}

Dieser Stoff hat die Eigenschaften eines Alkohols; denn er ist oxydierbar durch Permanganat, liefert einen Essigsäureester, welcher gegen Permanganat beständig ist, und einen o-Nitrobenzoesäureester, Schmelzp. 135 bis 
$137^{\circ}$. Das Dihydroolibanol ist nicht identisch mit dem Dihydroverbenol von Blumann und Zeitschel, welches ähnlich gewonnen wird, der gleichen Summenformel entspricht, aber fest ist und bei $58^{\circ}$ schmilzt.

Ein Versuch, $\beta$-Olibanol auf dieselbe Art zu reduzieren, zeigte, da $\beta$ hierbei neben harzigen Produkten nur unverändertes $\beta$-Olibanol wiedergewonnen wurde.

$0,1309 \mathrm{~g}$ gaben $0,3797 \mathrm{CO}_{2}$ und $0,1180 \mathrm{H}_{2} \mathrm{O}$.

$\begin{array}{ccc} & \text { Ber. für } \mathrm{C}_{10} \mathrm{H}_{16} \mathrm{O} & \text { Gef. } \\ \mathrm{C} & 78,88 & 79,13 \\ \mathrm{H} & 10,60 & 10,00\end{array}$

Dies Ergebnis ist insofern von Bedeutung, als es zeigt, daß $\beta$-Olibanol mit Dihydroverbenon und auch wohl mit Verbenol nicht identisch sein kann. Demnach würden für diesen Stoff nur noch die obigen Formeln II, IV und IV a übrigbleiben.

Um mehr Ausgangsmaterial zu erhalten, erbaten und erhielten wir von der Firma Schimmel \& Co. eine neue Menge Olibanumöl, diesesmal jedoch terpenhaltiges. Als dies Präparat der Destillation in vacuo unterworfen warde, erhielt man wieder einen Stoff, Siedep. ${ }_{15} 114$ bis $116^{0}, \mathrm{D}_{18} 0,9502$ von der Zusammensetzung $\mathrm{C}_{10} \mathrm{H}_{18} \mathrm{O}$.

0,1122 g gaben $0,3231 \mathrm{CO}_{2}$ und $0,1055 \mathrm{H}_{2} \mathrm{O}$.

$\begin{array}{ccc} & \text { Ber. für } \mathrm{C}_{10} \mathrm{H}_{16} \mathrm{O} & \text { Gef. } \\ \mathrm{C} & \mathbf{7 8 , 8 8} & 78,56 \\ \mathrm{H} & 10,60 & 10,52\end{array}$

Dieser Stofi ist aber leider nicht identisch mit dem $\alpha$-Olibanol; denn er läßt sich nicht wie dieses reduzieren und liefert beim Erhitzen über seinen Siedepunkt kein $\beta$-Olibanol, das zu Pinononsäure oxydiert werden kann. Der neue Stoff mag darum $\gamma$-Olibanol heißen. Er liefert bei der Oxydation eine flüssige Sänre und reagiert nicht mit Semicarbazid.

In diesem Stadium wurde die Untersuchung durch den Krieg unterbrochen. Das Weihrauchöl, welches das $\gamma$-Olibanol geliefert hatte, stand fünf Jahre lang in geschlossenem Gefäbe. Nach dieser Frist wurde es aber- 
mals untersucht, um festzustellen, ob sich nicht etwa in dem Öle beim Altern $\alpha$-Olibanol gebildet hatte; diese Untersuchung verlief ohne das genannte Ergebnis, es war auch nach fünf Jahren keine Spur davon gebildet. Es dürfte jetzt sehr schwer sein, neue Mengen Weihrauchöl zu erhalten; sobald das gelungen sein wird, soll die Untersuchung fortgesetzt werden. Sie wurde mit Mitteln der Wissenschaftlichen Gesellschaft von Freiburg i. B., welcher auch an dieser Stelle für die Unterstïtzung gedankt sei, durchgeführt. 